\title{
Religion(s) et école(s) en Israël
}

Religion(s) and school(s) in Israel

Religión(es) y escuela(s) en Israel

Denis Charbit et Myriam Darmoni

\section{CpenEdition}

\section{Journals}

Édition électronique

URL : http://journals.openedition.org/ries/1494

DOI : 10.4000/ries. 1494

ISSN : 2261-4265

Éditeur

Centre international d'études pédagogiques

Édition imprimée

Date de publication : 1 septembre 2004

Pagination : 85-100

ISBN : 978-2-85420-561-8

ISSN : 1254-4590

Référence électronique

Denis Charbit et Myriam Darmoni, «Religion(s) et école(s) en Israël », Revue internationale d'éducation de Sèvres [En ligne], 36 | septembre 2004, mis en ligne le 18 novembre 2011, consulté le 20 avril 2019 URL : http://journals.openedition.org/ries/1494; DOI : 10.4000/ries.1494 


\section{Religion(s) et école(s) en Israël}

\section{Denis Charbit Myriam Darmoni}

Dans tous les pays démocratiques, notamment en Europe, la division principale que l'on constate sur le plan institutionnel oppose l'École publique à l'École privée. Cette division, qui est liée à l'origine du financement (privé ou public) recoupe le plus souvent une problématique confessionnelle: les écoles privées ont le plus souvent pour vocation de dispenser un enseignement religieux spécifique pour les enfants de familles qui tiennent à transmettre, par le biais de l'École, une identité confessionnelle que l'École publique, de par sa neutralité, ne peut elle-même assurer. Cette distinction public/privé existe, bien sûr, en Israël, mais elle est loin de résumer et d'épuiser à elle seule la question de la religion à l'École qui est infiniment plus complexe. En effet il n'y pas, comme c'est le cas en France, à côté d'une École privée confessionnelle, une École publique qui serait laïque, au sens où elle serait neutre et indifférente à l'égard de toutes les religions au point de les exclure purement et simplement de l'espace scolaire et de l'enseignement. Si la plupart des écoles privées sont, en Israël, confessionnelles ${ }^{1}$, l'École publique se subdivise elle-même en plusieurs secteurs dont aucun, d'ailleurs, n'est strictement laïc si l'on entend par laïcité l'exclusion absolue de toute référence à un contenu relevant d'une religion. Le ministère de l'Éducation regroupe en son sein la gestion pédagogique et le financement de trois secteurs publics dont la constitution distincte s'explique, directement ou indirectement, par des raisons relatives à la place de la religion dans l'École :

- un secteur arabe dans lequel est dispensé un enseignement de l'islam;

- un secteur hébraïque destiné aux enfants de familles juives religieuses ou traditionnalistes incluant une pratique, un enseignement de la religion juive à part égale avec l'apprentissage des matières séculières ;

- un secteur hébraïque destiné aux enfants des familles juives agnostiques ou athées dans lequel l'étude de la Bible constitue une matière obligatoire enseignée au titre de patrimoine culturel, historique et littéraire et non comme un contenu de foi.

À ces trois secteurs intégralement subventionnés par l'État, il faut ajouter un quatrième intitulé «secteur reconnu mais non officiel» que l'État

\footnotetext{
1. Si les écoles privées étaient toutes confessionnnelles jusqu'aux années 1990, on assiste depuis à un épanouissement sans précédent d'établissements scolaires également privés à vocation thématique (axés, le plus souvent sur les arts ou une pédagogie active de la démocratie) créés par des collectifs de parents d'élèves instatisfaits de l'offre scolaire du secteur public.
} 
prend partiellement en charge jusqu'à un plafond de $60 \%$ des dépenses. Toutes les institutions fréquentées par les enfants de milieu ultra-orthodoxe sont soumises à ce régime, de même que les écoles chrétiennes.

«La religion et l'École». Il serait plus adéquat de parler de «religions» et d' "écoles», compte tenu des diverses confessions pratiquées et des types d'écoles constitutifs du paysage scolaire israélien.

Dans une première partie, nous verrons comment l'Éducation nationale a géré institutionnellement le pluralisme confessionnel du corps social israélien, étant donné que cette vision (occidentale) de la religion comme affaire personnelle et individuelle relevant de la conscience intime du croyant n'a pas cours, ou plus exactement, n'est pas majoritaire. Dans une seconde partie, nous aurons à cœur de montrer les évolutions les plus récentes qui témoignent de ce dynamisme et qui ont généré, ces dernières années, dans un contexte de libéralisme économique et de disparités sociales grandissantes, des visions de l'École plus composites, moins dichotomiques, qui contribuent à morceler un peu plus un tableau déjà bien divisé.

\section{L'ÉCOLE ARABE ET LA PLACE DE L'ISLAM}

S'il y a en Israël une École arabe dont la langue d'instruction exclusive est l'arabe et une École hébraïque, dont la langue d'instruction exclusive est l'hébreu, la distinction entre les deux types d'établissement ne se limite pas à la dimension linguistique. Elle a des effets directs sur le calendrier scolaire, lequel est fixé en fonction des traditions religieuses de chacune des communautés concernées. Si la rentrée a lieu uniformément au début du mois de septembre et que l'année s'achève pour tous à la fin du mois de juin, en revanche, entre ces deux dates, le déroulement n'est pas identique : le jour de repos hebdomadaire est le vendredi dans le premier cas, le samedi dans le second, à quoi il faut ajouter les congés scolaires qui correspondent aux fêtes religieuses juives et musulmanes qui rarement coïncident les unes avec les autres.

Cette séparation institutionnelle tient d'abord à la pluralité ethnoculturelle, linguistique et confessionnelle de la population israélienne ${ }^{2}$. Celle-ci est, en effet, composée d'une majorité juive de langue hébraïque et de confession juive d'une part et d'une minorité de langue arabe à dominante musulmane d'autre part (actuellemement $80 \%$ de musulmans, $8 \%$ de Druzes et $12 \%$ relevant des différentes églises chrétiennes d'Orient - catholiques romains, maronites, melkites, protestants).

2. La distinction n'est pas seulement linguistique, mais aussi ethno-culturelle et confessionnelle. Un nouvel immigrant juif issu d'un pays arabe et dont la langue maternelle est arabe est bien évidemment orienté vers une école hébraïque. 
Les distinctions confessionnelles n'ont pas créé mais renforcé un clivage premier d'ordre ethno-national qui avait conduit ces deux groupes à concevoir des projets politiques mimétiques sanctionnés en 1948 par une guerre gagnée par l'État d'Israël. Des sociétés fragmentées de la sorte cohabitant sur un même territoire peuvent, en théorie, se doter d'un projet unitaire d'intégration visant à transcender la distinction en vue de créer une culture commune, ce qui implique nécessairement l'hégémonie de la langue et de la culture majoritaire aux dépens de celle de la minorité. Ou bien l'on met en place un système qui offre à la minorité la reconnaissance de sa spécificité linguistique, culturelle et religieuse. C'est à ce choix que s'est rangé le gouvernement israélien depuis la création de l'État en proclamant le caractère officiel de la langue arabe au même titre que la langue hébraïque. Même s'il l'a fait sans consulter explicitement la population arabe par l'intermédiaire de ses représentants élus, il va de soi que cette deuxième option correspondait aux vœux de la population concernée. Toutefois, pour des raisons liées à l'absence d'un règlement définitif du contentieux israélo-arabe qui aurait apaisé, sinon dissipé, les méfiances réciproques, le gouvernement a décidé de respecter cette réalité culturelle, linguistique et religieuse tout en se gardant bien d'octroyer aux Arabes une autonomie effective de décision et de gestion. Autrement dit, l'École publique en secteur arabe a été étroitement contrôlée par le ministère, maintenue dans un état de dépendance institutionnelle, ce qui s'est longtemps traduit par le fait que la direction et l'inspection relatives au secteur arabe ainsi que les commissions pédagogiques chargées d'établir les programmes étaient majoritairement composées de juifs ${ }^{3}$. Cependant, les graves inégalités entre secteur juif et arabe en matière de moyens investis, de formation des professeurs, et de taux de réussite au baccalauréat, régulièrement dénoncées par les rapports du contrôleur de l'État, et récement par la commission Orr, ne peuvent éluder la «révolution scolaire» intervenue en milieu arabe depuis la création de l'État d'Israël : 6780 lycéens répartis dans 46 institutions scolaires en 1948, 235557 élèves dans 447 établissements en 1991, 381000 en 2002. Pour avoir une échelle de comparaison, dans la même période, la population arabe s'est multipliée par 5,5, passant de 156000 à 875000 habitants, tandis que la population lycéenne l'a été par 35,5, ce qui a entraîné une chute considérable de l'illettrisme, transformé la structure sociale en fondant l'autorité sur le mérite, non sur la tradition et l'âge.

Alors que dans la loi sur l'Éducation nationale votée en 1953, les objectifs pédagogiques furent rédigés de manière uniforme sans spécifier des finalités propres à la communauté arabe, leur révision effectuée en 1980 en tint compte : l'objectif de l'École publique en secteur arabe est «de baser l'éducation sur les

3. Cette situation a nettement changé depuis une quinzaine d'années. À l'heure actuelle, si la situation ne peut être définie en terme d'autonomie en matière d'éducation (ce qui réveille toujours dans l'opinion juive la crainte d'une revendication irrédentiste ou indépendantiste), la participation de professeurs, d'inspecteurs et d'universitaires arabes dans les instances de décision relatives à l'éducation en secteur arabe s'est considérablement accrue. 
fondements de la culture arabe; sur les acquis de la science; sur l'aspiration à la paix entre Israël et ses voisins; sur l'amour de la patrie commun à tous les citoyens de l'État et la loyauté envers l'État d'Israël - tout en mettant l'accent sur les intérêts communs et la promotion de la spécificité communautaire des Arabes israéliens et la connaissance de la culture juive ${ }^{4}$. L'islam n'est pas explicitement mentionné, mais il est sous-entendu par la référence à la culture arabe dont l'une des dimensions principales est d'ordre religieux. En vérité, la revendication qui tient le plus à cœur à la population arabe est la reconnaissance par les autorités israéliennes de son identité palestinienne spécifique, bien plus que son appartenance générale à la nation arabe et à l'islam qui les dissout dans un grand ensemble dont elle ne serait qu'un appendice ${ }^{5}$.

En théorie, l'École arabe est multi-confessionnelle puisqu'elle rassemble sous le même toit linguistique, musulmans, druzes et chrétiens. En pratique, cette cohabitation est très rare. Les Druzes vivent dans leurs propres communes et ont donc leurs propres écoles. Depuis 1978, un secteur druze, distinct du secteur arabe, a été créé. Pour ce qui est des Arabes chrétiens, si on les trouve en très petit nombre dans les écoles publiques arabes, c'est que, pour des raisons de reproduction culturelle et sociale, leurs parents, qui appartiennent à la bourgeoisie aisée, préfèrent envoyer leurs enfants dans les écoles chrétiennes extrêmement sélectives. À cet égard, à cause du sous-développement en matière d'équipement et d'innovation pédagogique, il est fréquent de voir la bourgeoisie arabo-musulmane faire le même choix d'envoyer ses enfants, surtout s'ils sont doués, dans des écoles chrétiennes.

Contrairement à l'école hébraïque divisée en secteur religieux et séculier, une subdivision de ce type n'existe pas en secteur arabe ni pour les Druzes ni pour les musulmans. Pour les premiers, il n'y a pas à proprement parler de cours d'instruction religieuse, du fait que les fondements de la secte druze (dissidence de l'islam qui l'a longtemps pourchassé) doivent rester secrets et n'être connus que des sages de la communauté. En revanche, cet interdit n'étant pas de règle dans l'islam, des cours d'instruction religieuse musulmane sont dispensés à l'école primaire, puis au collège et enfin au lycée (en classe de seconde, principalement en section lettres et histoire, à raison de trois heures par semaine). À la fin de la seconde, les élèves peuvent présenter cette matière au baccalauréat. Toutefois, peu de candidats choisissent cette option ${ }^{6}$. Le programme tourne autour des fondements de la foi: le Coran, lecture et

4. Cité par Sammy Smookha, The Orientation and Politicization of the Arab Minority in Israel, Monograph Series on the Middle East, n.2, 1984, p. 68.

5. La commission chargée de réviser les objectifs pédagogiques du programme d'histoire pour l'enseignement secondaire définis, la première fois, en 1982 a stipulé comme but celui « d'approfondir le sentiment d'appartenance au peuple arabe palestinien, à la nation arabe et sa culture, à l'État d'Israël et ses citoyens ».

6. Les élèves d'origine chrétienne sont évidemement dispensés de ces cours et peuvent sortir de classe. Ils sont en général trop peu nombreux pour qu'on leur assure un cours d'instruction chrétienne analogue. 
commentaire; la charia; l'histoire de Mahomet le prophète; l'essor de l'islam et de la civilisation musulmane dans l'histoire. Il s'agit moins de former à la foi que de transmettre une connaissance livresque d'un pilier essentiel de la culture arabe articulée à une tradition qui se pratique au sein de la famille. Toutefois, la connaissance de l'islam n'est qu'un élément d'un ensemble plus vaste relatif à la culture arabe, incluant la littérature et l'histoire ancienne et contemporaine $\mathrm{du}$ monde arabe. Les enseignants, qui sont généralement des professeurs d'histoire, se gardent bien de parler de l'islam politique actuel.

Contrairement aux élèves juifs qui ignorent tout de l'islam comme fait religieux, les élèves arabes acquièrent quelques notions de judaïsme dans le cadre de leur apprentissage de la langue et de la culture hébraïques ${ }^{7}$.

Dans quelle mesure l'islamisation progressive de la population palestinienne en Israël se manifeste-t-elle sur le plan scolaire? Cette évolution est liée à la participation du Mouvement islamique aux élections municipales. Au début de cette phase, il n'était pas rare que, dans certains villages, des militants proposent aux jeunes adolescents une formation religieuse après l'école, dans les mosquées ou d'autres lieux mis à leur disposition par les autorités municipales, arguant de son caractère pur et authentique, comparée à la version frelatée et partielle proposée par l'enseignement public sous le contrôle de l'État juif. Depuis, le mouvement islamique, récoltant les fruits de son militantisme et de ses succès électoraux, poursuit son action à l'intérieur des établissements en invitant ses membres à entrer dans les comités de parents d'élèves pour faire valoir des revendications d'ordre religieux. Ainsi, alors que, traditionnellement, les écoles arabes sont mixtes, on assiste, dans les localités où la proportion de pratiquants est en hausse, à l'ouverture de classes réparties selon les sexes. Sans que la décision soit imposée à la majorité, les jeunes filles sont tenues de porter des vêtements décents : pantalon long et chemise à manches longues et, bien sûr, hidjeb ${ }^{8}$.

À ce propos, une affaire autour du «voile islamique» a eu lieu également en Israël, mais outre qu'elle n'a pas suscité autant de remous, il est intéressant de voir comment les différences de normes et de système ont entraîné une gestion et une décision tout à fait opposée à ce qui s'est passé en France. En 1990, Mona Jabreen, une jeune lycéenne musulmane, informée par la direction de l'école chrétienne privée de Saint-Joseph à Nazareth, où elle avait été

7. Cette situation illustre l'asymétrie de leur condition respective. Alors que l'hébreu est obligatoire en secteur arabe à partir de huit ans, de telle sorte qu'au bout de neuf ans d'études, un jeune Arabe obtient la maîtrise d'une compétence linguistique susceptible de favoriser ses facultés de communication avec la majorité hébraïque du pays, l'inverse n'est pas vrai : dans l'école hébraïque, l'arabe n'est que la troisième langue optionnelle dispensée à partir de la sixième. Elle vient après l'anglais qui est obligatoire dès l'âge de huit ans. Toutefois, de toutes les troisièmes langues proposées en option aux élèves, l'arabe arrive très largement en tête.

8. Elie Rekhes, The Arab Minority in Israel : Between Communism and Arab Nationalism, 1965-1991, Moshe Dayan Center, Tel Aviv University, Hakibboutz Hameouchad, coll. «Kav Adom », 1993, p. 153. 
acceptée pour ses brillants résultats à l'école primaire, qu'elle ne pourrait se rendre en classe le visage voilé ni être dispensée des excursions et des cours de gymnastique, décida de porter l'affaire devant la Cour suprême, invoquant les principes de liberté religieuse et d'égalité. L'arrêt rendu par la Cour fut sans équivoque: "attendu que l'uniformité exigée par l'établissement relève de considérations pédagogiques découlant du caractère et de l'essence même dudit établissement», elle estima que si l'école publique est, quant à elle, tenue de respecter intra muros les coutumes et les signes religieux de toute confession sans discrimination, en revanche, le secteur privé, de par sa nature religieuse spécifique, est en droit d'établir ses règles de conduite et de les imposer aux impétrants, puisque ceux-ci ne sont pas obligés de s'y inscrire. Et c'est ainsi que, sous la recommandation de ses parents, elle préféra se rendre dans une école publique israélienne puisqu'elle pouvait y porter librement le foulard de son choix. En cette affaire, on le voit, la règle israélienne est l'inverse de celle qui prévaut désormais en France: c'est l'école publique qui a, en Israël, le devoir d'accueillir une jeune musulmane désireuse de garder le voile; c'est, en France, l'école privée qui pourra le faire ${ }^{9}$.

Ces écoles privées sont en général associées à des ordres religieux dont le siège principal est hors d'Israël; le personnel enseignant est mixte (musulman ou chrétien). L'État d'Israël couvre une partie de leur budget et leur programme est aligné sur les exigences du ministère. Certaines de ces écoles jouissent d'une excellente réputation et obtiennent même le record national au baccalauréat ${ }^{10}$ (plus de $90 \%$ de réussite). Creuset de la formation des élites arabes, la bourgeoisie musulmane n'hésite guère à y envoyer ses enfants, au point que les effectifs indiquent aujourd'hui une majorité d'élèves musulmans (65\% des élèves). De ce fait, ou bien on a purement et simplement aboli les cours d'instruction religieuse dans ces établissements devenus, de fait, pluri-confessionnels, ou bien on les maintient, offrant alors aux élèves musulmans une dispense. En revanche, on y sanctionne sévèrement toute infraction à la tolérance inter-religieuse : un élève qui a insulté un condisciple membre d'une autre confession est définitivement exclu de l'école s'il récidive. Les mœurs internes en vigueur témoignent bien de l'ancrage conservateur : l'uniforme est de règle en classe; toute fête organisée par les élèves exige la présence d'un professeur.

Il peut arriver, surtout dans les villes mixtes, que des écoliers arabes accomplissent leur scolarité dans une école hébraïque, les parents jugeant que cette dernière est d'un niveau supérieur à l'école arabe locale et qu'elle facilitera

9. Yaïr Ettinger, «Au lieu du hijeb, les infirmières musulmanes porteront le couvre-chef vert en vigueur dans les salles d'opération », Haaretz, 5 avril 2004.

10. L'école des sœurs de Nazareth affiche un taux record de $98 \%$ de réussite au baccalauréat, $95 \%$ pour l'école grecque de Haifa, par rapport à la moyenne nationale qui atteint péniblement les 52,8\%.70\% des élèves issus de l'école grecque depuis sa fondation en 1952 ont poursuivi, par la suite, des études supérieures. Voir David Retner, "C'est là que l'on forme l'élite», Haaretz 25/5/2004, p. 42. 
donc une meilleure intégration sociale. Le phénomène est devenu, ces dernières années, de plus en plus rare. La socialisation de l'enfant dans sa communauté et son milieu d'origine est considérée, en Israël, comme un facteur de sécurité propice à la structuration de sa personnalité, et l'emporte, de ce fait, sur des perspectives de mobilité sociale.

\section{L’ÉCOLE HÉBRAÏQUE, SA FONCTION ET SON HISTOIRE}

À l'époque du yichouv, c'est-à-dire de la nouvelle communauté juive organisée en Palestine, les partis politiques jouèrent un rôle essentiel dans la préfiguration de l'État, fournissant à leur clientèle l'encadrement social et institutionnel nécessaire à leurs besoins. C'est la raison pour laquelle les grandes formations politiques se dotèrent d'un réseau scolaire propre, lequel constituait pour les élèves une structure de socialisation des plus efficaces. Au-delà de la pression exercée par ces forces centrifuges en l'absence d'un État régulateur, cette division (ou ce pluralisme) illustre bien le caractère total et entier de ces orientations idéologiques à l'intérieur comme à l'extérieur du mouvement sioniste : à chacune sa vision du pays à naître et, en conséquence, à chacune ses principes d'éducation, ses valeurs pédagogiques et ses contenus programmatiques spécifiques. Il n'y eut pas moins de quatre réseaux entre lesquels on choisissait en fonction d'une affiliation idéologique et/ou religieuse : un réseau sioniste-socialiste appelé le «courant des travailleurs»; un réseau généraliste qui dépendait du parti libéral et bourgeois des «sionistes généraux»; un réseau sioniste-religieux pour le parti Mizrachi qui avait fondé son identité idéologique sur la synthèse du sionisme et du judaïsme religieux; enfin, un réseau ultraorthodoxe émanant de l'Agoudat Israël destiné à la communauté de stricte observance hostile au sionisme. À la veille de la proclamation de l'État d'Israël, ne voulant se priver d'aucun concours et souhaitant rallier les partis religieux, y compris les formations antisionistes, David Ben-Gourion, en tant que leader de l'Agence juive, l'appareil exécutif du yichouv, avait consenti à proposer un compromis historique relatif aux rapports qui seraient noués entre la Synagogue et l'État ${ }^{11}$. Concernant la question scolaire, il prit l'engagement d'assurer une totale indépendance au secteur orthodoxe. Le compromis n'était pas mince car Ben-Gourion n'avait pas caché son grand dessein étatique et centralisateur : après cette période durant laquelle les grands courants idéologiques du yichouv avaient créé leurs propres institutions ( $\mathrm{y}$ compris des organisations para-militaires, par exemple), l'heure était venue, avec l'avènement de l'État, de nationaliser, d'unifier, d'étatiser, de créer, pour chacun des domaines exigeant l'autorité de l'État, un grand service 
public et notamment, une armée (Tsahal) et une École. L'enjeu était particulièrement important car, entre 1948 et 1953, la population israélienne avait triplé avec l'arrivée massive de centaines de milliers de juifs venus d'Europe orientale ou des pays arabo-musulmans (en particulier, l'Irak, le Yémen et le Maroc), ou encore des rescapés des camps de la mort, qu'il fallait intégrer non seulement sur le plan social et dans le marché du travail, mais encore hébraïser sur le plan linguistique et culturel. La première mission assignée à l'Éducation nationale était, comme son nom l'indique, de faire de l'École le creuset indispensable de l'édification de la nation, d'où l'instauration dès 1949, alors même que les structures et les financements étaient inexistants, d'une École obligatoire et gratuite de six à treize ans. Quatre ans plus tard, Ben-Gourion voulut mettre de l'ordre dans ce système scolaire hérité de la période du Mandat britannique et toujours éclaté en quatre courants. Votée le 12 août 1953, la loi décréta la mise en place d'une École publique unifiée en lieu et place des courants autonomes qui avaient prévalu jusque-là. Elle fait référence à la subdivision en un secteur étatique et un secteur étatique-religieux, ce dernier étant identique au premier en ce qui concerne les disciplines profanes auxquelles s'additionnent des contenus religieux complémentaires. En fait, Ben-Gourion eut à composer avec les revendications des partis religieux déterminés à préserver la singularité de leur École. Le courant ultra-orthodoxe était désormais un organisme défini comme «reconnu mais non-officiel» : en d'autres termes, l'État consentait à subventionner le secteur orthodoxe sans obtenir en contrepartie un droit de regard substantiel sur les programmes et sur la formation des maîtres. De la sorte, le réseau ultra-othodoxe maintenait son autonomie externe, hors État, vis-à-vis des pouvoirs publics. Compte tenu de son approbation du sionisme et de la création de l'État d'Israël, le parti national religieux souhaitait, lui, que son réseau soit public tout en continuant de jouir d'une autonomie, interne cette fois, dans l'État: le réseau fut officiellement reconnu et désigné comme «École publique-religieuse» et une direction pour l'éducation religieuse fonctionnant comme une sorte d'enclave, disposant de son propre institut de formation des maîtres, fut établie au sein même du ministère de l'Éducation. En dépit des intentions centralisatrices de Ben-Gourion, les réseaux religieux avaient su habilement tirer leur épingle du jeu et la loi n'avait, en réalité, procédé qu'à la fusion des deux courants d'obédience laïque. Plusieurs députés libéraux et travaillistes crièrent à la discrimination et s'opposèrent à cette concession dont les réseaux scolaires religieux étaient les seuls bénéficiaires. La loi fut votée grâce à une courte majorité mais Ben-Gourion pouvait savourer son triomphe: il avait fait l'économie d'une Kulturkampf, et pariait sur le fait qu'en terme d'effectifs, le secteur orthodoxe et le secteur national religieux attireraient, à eux deux, une quantité d'élèves qui irait en diminuant. Autrement dit, globalement, la plus grande partie de la jeunesse juive passerait par le rouleau compresseur d'un système d'éducation nationale unifié visant à transformer les sabras (nés en Israël) et les jeunes juifs venus des quatre coins du monde en Israéliens conscients de leur patrimoine et disposés à relever les défis d'une nation à reconstruire et à réunifier. 


\section{L'ÉCOLE PUBLIQUE EST-ELLE LAÏQUE?}

L'école est publique, mais peut-on dire qu'elle est laïque? Pour répondre avec précision, il importe de s'entendre sur ce que «laïcité » veut dire. Le modèle de l'école publique israélienne est laïc au moins en deux sens qui relèvent du modèle français : les membres du clergé juif, autrement dit les rabbins, ne peuvent y enseigner. Certes, il n'y a pas d'interdiction formelle mais c'est une convention totalement respectée par les directeurs d'établissement qui, s'ils contrevenaient à cette règle, trahiraient, en quelque sorte, un contrat tacite passé avec les parents d'élèves. On peut également dire que l'école est laïque en ce sens que les élèves se déclarent, dans leur écrasante majorité, agnostiques, athées ou indifférents à la chose religieuse. Certes, il peut y avoir parmi les élèves des traditionnalistes, autrement dit des enfants qui observent certaines coutumes dans le cadre familial. À l'intérieur de l'école, il n'y a pas de lieu réservé à la prière. Tout au plus y a-t-il, à l'entrée de chaque classe, une mezouza encore que celle-ci est en matière plastique et surtout vidée du parchemin qu'elle est censée abriter pour être conforme à la prescription religieuse. Reste la question, somme toute essentielle, du programme. Si la religion juive est dispensée à l'école publique, c'est par le biais de l'enseignement de la Bible et plus précisément, l'étude de ce que l'on appelle communément l'Ancien Testament. En ce sens, la laïcité à l'israélienne ne correspond à aucune des deux définitions admises en France: ni celle de la neutralité qui exclut tout enseignement des religions; ni celle de la laïcité de combat qui dispense, par le biais du cours de philosophie et le discours de la raison une alternative à la tradition. Une définition stricte, une école laïque est tenue d'exclure tout ce qui relève d'une religion, l'école publique hébraïque ne mérite pas non plus d'être désignée comme telle. En vérité, le programme "religieux» se limite à l'étude de la Bible, en tant que pièce maîtresse du patrimoine culturel national. C'est une matière obligatoire et tout élève est tenu de la présenter au baccalauréat. Toutefois, et la précision est capitale, la Bible n'est nullement enseignée comme un contenu de foi, mais comme l'histoire ancienne du peuple juif. Autrement dit, il s'agit là d'un enseignement qui, délibérément, sécularise le texte biblique et, sciemment, en évacue la portée religieuse. Les Psaumes du roi David ne sont pas étudiés pour louer Dieu, mais pour dégager la perfection de l'art poétique du poète-roi; le Livre des Rois est traité comme un récit historique qui relate des faits, des épopées, des aventures dont on ne dissimule pas l'aspect mythique et le caractère légendaire qu'ils peuvent avoir; parce que l'action se déroule en terre d'Israël, la Bible atteste ainsi du lien inaliénable du peuple à sa terre. Des questions morales peuvent être soulevées à propos de l'épisode du sacrifice d'Isaac ou des souffrances de Job mais l'on s'applique à dissocier tous ces aspects de leur signification proprement religieuse. Toutefois, cet enseignement fut, pour beaucoup, un moyen de découvrir la terre d'Israël la Bible à la main, de découvrir les richesses de la langue 
hébraïque dont la Bible est le chef d'œuvre inégalé, de se reconstituer une généalogie qui ne plonge pas ses racines dans les pays de la diaspora de leurs parents ou de leurs grands parents mais dans ce pays même et avec les personnages de ce panthéon retrouvé et reconstitué. La Bible est livre d'histoire et manuel de géographie et aussi dictionnaire de la langue hébraïque. Elle fut, à bien des égards, une œuvre édifiante mais dans une perspective qui n'avait rien de religieux proprement dit, d'autant que cette histoire est plus le récit des infidélités du peuple juif à son Dieu qu'une imitation de Moïse ou d'Abraham. Cependant, cette instrumentalisation de la Bible qui fut, à bien des égards, une remarquable entreprise de sécularisation, est aujourd'hui - depuis trois décennies au moins tout à fait dépassée. Cela est dû à deux évolutions qui ont considérablement affecté son statut. D'une part, des changements idéologiques - qu'on les appelle individualisme, société de consommation ou américanisation - sont intervenus à partir des années soixante-dix et ont eu pour effet de dévaloriser cet enseignement de la Bible ainsi que celui de l'histoire juive ou de la littérature hébraïque lesquels, pris comme un tout, avaient pour vocation de construire l'identité nationale du nouvel homme juif. C'est en effet, comme l'a noté Daniel Elazar, une conception plus fonctionnelle et instrumentale de ces matières qui a fini par prévaloir en Israël. Autrement dit, l'école est moins conçue comme une cellule d'intrégration culturelle mais comme une structure de préparation sinon de formation à la vie active : ce sont donc les mathématiques, l'anglais, les sciences exactes qui l'ont emporté au détriment des humanités juives ${ }^{12}$. De plus, c'est la formation même des professeurs de Tanakh (initiales de la Bible en hébreu) qui s'est profondément transformée. Au plaisir du texte qui était souvent l'aiguillon principal de l'enseignant, s'est substitué un appareil critique de concepts et de théories formelles que l'élève doit apprendre à manier, ruinant ainsi toute chance d'être touché, interpellé par la parole biblique. De moins en moins d'étudiants laïcs choisissent les études bibliques à l'université et les directeurs d'école sont parfois contraints de faire appel à des enseignants religieux. Enfin, il est indéniable que pour les jeunes, l'étude d'un texte sacré où Dieu se manifeste à chaque instant apparaît comme une contradiction flagrante avec leur athéisme ou leur indifférence religieuse. Le résultat est que la Bible n'est plus aujourd'hui ce vecteur d'identité nationale qu'elle avait été si longtemps. Elle devient, pour l'élève, une matière qui rebute, un archïsme qui inspire l'ennui et l'incompréhension et dont les problématiques sont à mille lieux de leur univers colonisé par la télévision, la vidéo, Internet et les DVD, game boy et autres playstations. De telle sorte que si la religion entre par la fenêtre de ce domaine biblique qu'on lui réserve, la tendance anti-cléricale dominante dans la population israélienne fait vite écran pour empêcher une curiosité intellectuelle et spirituelle.

12. Daniel J. Elazar, «Education in a Society as a Crossroads: An Historical Perspective of Israeli Education», Israel Studies, vol. 2, n. 2, Fall, 1997, p. 52. 


\section{L'École PUBLIQUe-RELIGIEUSE, ACQUIS ET LIMITES}

L'école publique-religieuse, elle non plus, n'a pas été épargnée par une évolution sociale et idéologique qui l'affecte aujourd'hui. Avant d'en discerner les causes et les effets, décrivons ce par quoi elle peut être définie, à juste titre, comme religieuse, en commençant par indiquer ce qui a fait sa spécificité, et même son originalité : inspirée de l'idéologie sioniste-religieuse du Mizrachi, l'École a été bâtie sur cette idée éminemment audacieuse, dans le monde religieux, qu'en matière d'éducation, la distinction cognitive et non native entre matières sacrées (limoudei kodesh) et matières profanes (limoudei hol) n'implique pas que la formation scolaire doit être toute entière orientée vers les premières au détriment des secondes réduites aux connaissances élémentaires. L'objectif consistait à pratiquer l'une et l'autre discipline, car il n'y a de sacré que si l'on reconnait le profane et qu'on le valorise plutôt que l'abolir. Cet esprit de synthèse est particulièrement visible dans les devises du Mizrachi empruntées à la sagesse juive : «loi et travail (Thora Ve-Avoda) ou encore ce célèbre dicton «il n'y a pas d'étude sans farine ni de farine sans étude» qui enjoint à l'homme de subvenir à ses besoins pour pouvoir se livrer à l'étude de la Loi. Complémentarité et compatibilité sont de mise et les écoles religieuses se voulaient, en ce sens, une voie médiane se gardant bien de refouler le sacré à l'instar de l'école laïque ou de refouler le profane à l'instar de l'institution ultraorthodoxe. Outre le choix du sionisme et la reconnaissance de l'État d'Israël, il est une autre nouveauté qu'a introduite l'école religieuse, c'est le droit des jeunes filles à bénéficier de l'instruction, c'est le principe de la mixité dans les classes. En aucun cas cependant cette reconnaissance de la valeur du travail, de la formation généraliste ou du principe de la mixité dans les classes ne devait altérer la visibilité du religieux dans l'établissement. Garçons et filles doivent porter les signes respectifs : un couvre-chef sur la tête (la kippa) et les tsitsit au bas de la chemise pour les premiers, jupes longues et chemises à manches longues en signe de pudeur pour les secondes (le pantalon est interdit); la place primordiale du religieux se traduit par l'office du matin qui précède l'étude, par un lieu spécial, un oratoire, affecté à la prière; par une bibliothèque religieuse richement fournie en plus d'une bibliothèque générale; par le personnel enseignant et administratif qui doit attester de sa foi et de sa pratique religieuses (les rabbins peuvent être invités à y enseigner); enfin par une part essentielle dévouée aux matières religieuses dans le cursus scolaire : étude de la Loi écrite et des livres de la Bible, d'une part, de la Loi orale (le Talmud) d'autre part, ainsi que l'étude des décrets halakhiques (dinim) qui régissent le comportement privé et public et le déroulement des fêtes religieuses. En revanche, les programmes scientifiques sont identiques à ce qui est enseigné dans l'école publique non-religieuse. Incontestablement, l'école religieuse a su produire des élites sociales qui se sont intégrées dans l'administration publique, dans 
l'armée, à l'université, en particulier l'université religieuse de Bar-Ilan. Toutefois, elle est plus une école de socialisation religieuse qu'une éducation à la foi. Le caractère orthopraxe du judaïsme incite à mettre l'accent sur l'accomplissement, juste et précis, du commandement, moins à se poser des questions sur la foi elle-même. Les élèves sont "religieux» par habitude et inertie, par «tradition» plus que par une quête spirituelle authentique. Le cadre offre une protection, une sécurité indéniables mais comme telle, l'école n'est pas un lieu propice à l'expérimentation religieuse.

Après avoir atteint son apogée dans les années quatre-vingt, l'école nationale-religieuse traverse actuellement une crise d'identité, liée à des évolutions qui, accomplies hors les murs, a fini par pénétrer l'enceinte de l'école pour la déstabiliser. Il y a, d'une part, l'importance qu'a revêtue, pour le sionisme national-religieux, la Yechivat Mercaz Harav ${ }^{13}$, laquelle a déplacé l'orientation moderniste mêlant vie pratique et vie spirituelle vers une version messianiste active du judaïsme qui s'est traduite par l'impératif de coloniser la JudéeSamarie. À l'école, cela s'est aussitôt traduit par le primat accordé à la valeur $\mathrm{d}^{\prime}$ «Eretz Israël ${ }^{14}$ au détriment du Livre et de la Loi. À cet égard, l'école parvient mieux à fabriquer des patriotes, des nationalistes ou des «faucons» en politique, de futurs soldats disposés à servir le pays qu'à former des consciences spirituelles morales. Le charisme des leaders, la certitude que leur action s'inscrit dans un plan divin a conduit à un dogmatisme idéologique, un conformisme religieux à la prépondérance des arguments d'autorité qui étouffent la liberté créative. D'autre part, alors que, pendant très longtemps, le sioniste religieux ne se sentait nullement intimidé par l'orthodoxie religieuse non-sioniste et arborait avec fierté la synthèse de tradition et modernité, il n'en est plus de même depuis plusieurs années, ce qui a conduit à la remise en cause de quelques-uns des aspects modernes, notamment la mixité. Alors que les écoles religieuses réunissaient filles et garcons adhérant, de surcroît, au même mouvement de jeunesse, aujourd'hui la tendance est à la surenchère en matière religieuse et à la séparation des sexes. Enfin, de plus en plus de familles traditionalistes, redoutant pour leurs enfants la permissivité qu'elles attribuent à l'école laïque et appréciant justement la discipline en vigueur dans les écoles religieuses, font le choix de l'école religieuse; mais les enfants n'étant pas réellement animés d'une véritable ferveur religieuse, c'est, dans certains établissements, le caractère de l'école qui s'en est trouvé affecté. La revalorisation de l'étude en yeshiva couplée avec un recrutement populaire de traditionalistes, lequel ne contribue pas à relever le niveau de l'école, a provoqué une hémorragie qui affecte durement l'avenir de l'école publique-religieuse et crée, pour ainsi dire, un système à deux vitesses : une école publique-religieuse pour les 
plus démunis sur le plan matériel et spirituel et d'autre part un système parallèle, élitiste, sélectif, idéologique et très coûteux les yeshivot (pour garçons) et les oulpanot (pour filles). Adolescents des deux sexes étudient jusqu'à treize heures les études dites sacrées, passant l'après midi jusqu'à dix-huit ou dix-neuf heures aux études profanes. Ces horaires prolongés nécessitent une participation des parents d'autant plus grande que les familles religieuses sont souvent nombreuses avec cinq enfants en moyenne.

Cependant, les élèves eux-mêmes secouent le système et apportent des revendications nouvelles qui témoignent d'une recherche de soi réconciliée harmonisée avec leur adhésion religieuse : une plus grande ouverture sur le monde, la prise en compte d'une certaine spiritualité post-moderne, moins d'études de guemara et des cours sur le hassidisme. Des prurits de consicence féministe traversent aussi l'école religieuse. Des sections cinéma et communication sont proposées dans le secondaire à la demande des élèves, des filières musique et danse également.

Paradoxalement, la place de la religion dans les deux modèles d'école est à l'origine de leur crise respective. Elle est plus grave pour l'école religieuse, car cette dernière voit ces effectifs fondre au profit des yeshivot sélectives. C'est parce que l'offre religieuse ne correspond plus à la demande que des contingents de sionistes religieux eux-mêmes formés dans ces écoles envoient leur progéniture dans ces yeshivot de très haut niveau. Cette revendication d'une excellence religieuse, d'un épanouissement dans l'étude ne serait-elle pas aussi un moyen, pour les parents, de contourner l'intégration scolaire avec des élèves en difficulté? Donc de rationaliser par une revendication d'apparence religieuse une volonté sélective?

Sans qu'il en soit de même sur le plan des effectifs, on assiste cependant à un phénomène de nature analogue dans l'école publique : à un autre niveau, des parents s'inquiètent également du caractère superficiel de l'identité juive transmise aux enfants et exigent le renforcement de ces matières. Tirant parti de la loi de 1953 qui autorisait les parents d'élèves à déterminer $25 \%$ du programme, des parents se sont organisés pour créer au sein de l'École publique un réseau d'établissements scolaires appropriés. Ces écoles dites Tali (acronyme de «renforcement des études juives») ont vocation à redonner à l'école sa vocation identitaire en plus de son objectif instrumental d'apprentissage. Cette quête d'une alternative n'est pas sans rapport avec une préoccupation spécifique à des parents d'élèves concernés et impliqués dans l'éducation de leurs enfants, appartenant à des couches aisées et détenteurs d'un bagage intellectuel audessus de la moyenne et qui souhaitent, par ce biais, éviter le nivellement par le bas auquel sont confrontées les deux écoles. Nonobstant cet aspect-là, ces expériences témoignent d'une volonté, que certains qualifient de post-moderne, de dépasser les visions dichotomiques école laïque/école religieuse en matière d'enseignement du judaïsme. Des écoles expérimentales ont été créées ces 
dernières années dans le but de faire étudier ensemble enfants de familles laïques et religieuses, côte à côte. Ces écoles expérimentales sont les laboratoires de tentatives courageuses pour penser et essayer d'articuler un mieux-vivre ensemble entre laïcs et religieux en Israël.

\section{ÉCOLES ULTRA ORTHODOXES : «SECTEUR RECONNU MAIS NON OFFICIEL "}

L'austérité économique a contribué à produire un autre cas de figure de plus en plus fréquent : des parents de familles modestes choisissent d'envoyer leurs enfants dans des écoles ultra-orthodoxes (comme celles du parti Shas). Ce n'est pas qu'ils soient devenus orthodoxes, ils sont le plus souvent traditionnalistes. S'ils y inscrivent leurs enfants, c'est qu'on y pratique la journée continue (jusqu'à seize heures au lieu de treize heures) et qu'on livre à l'enfant gratuitement un repas chaud et un transport aller et retour. Mais c'est la corde ethnicoculturelle, l'identité sépharade ou judéo-orientale que le Shas a fait vibrer en exaltant la splendeur passée au regard de la misère actuelle et en appelant les masses à se ressourcer aux origines (c'est, d'ailleurs, le nom du réseau scolaire "Vers la source», El Hamaayan). On doit tenir compte également du charisme des leaders, notamment des vieux sages, le grand-rabbin Ovadia Yossef et le rav kabbaliste Kadouri. Comme dans le système religieux décrit plus haut, on retrouve cette séparation institutionnelle en deux écoles, l'une destinée aux enfants de familles traditionalistes et le plus souvent largement ignorantes du judaïsme et l'autre, plus particulièrement destinée aux élèves qui veulent approfondir leurs connaissances religieuses.

Dans ces établissements, il convient non de discerner la place de la religion à l'école mais de dire, tout simplement, que la religion est l'école. Aucun enseignement profane significatif n'est dispensé. Les enfants, dès l'âge de trois ou quatre ans, apprennent à lire et se consacrent à l'Étude avec un E majuscule. Et c'est ensuite, successivement ou en parallèle, l'étude de la Bible, de la Michna, de la Guemara et du Talmud.

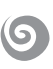

La pluralité, la diversité, pour ne pas dire l'hétérogénéité, des identités culturelles, des langues, des confessions, des convictions en matière religieuse (qui vont de l'ultra-athéisme à l'ultra-orthodoxie) est telle, que même si des choix significatifs dans tel ou tel sens sont opérés, Israël n'en apparaît pas moins comme un patchwork inachevé et dynamique en perpétuel processus de fabrication. Dans La Démocratie d'Israël, Claude Klein proposait de substituer à la désignation péjorative des divisions internes comme relevant d'une «société de 
clivages» le terme plus laudatif de «mosaïque sociale ${ }^{15}$. À cet égard, aussi centripète et centralisateur qu'ait voulu être Israël - et il l'a été incontestablement dans les premières décennies de son existence - on voit bien qu'il est tiraillé, harcelé, secoué par des tendances centrifuges qui émanent des composantes diverses de la société israélienne. L'examen du statut de la religion à l'École illustre parfaitement cette double vocation «juive et démocratique»par lequel l'État d'Israël est officiellement défini mais aussi ce dynamisme revendicatif en quête de nouvelles synthèses.

15. Claude Klein, La Démocratie d'Israël, Paris, Seuil, coll. Science politique, 1997. 
